doi: $10.13108 / 2021-13-4-123$

\title{
LAW OF LARGE NUMBERS FOR WEAKLY DEPENDENT RANDOM VARIABLES WITH VALUES IN $D[0,1]$
}

\author{
O.Sh. SHARIPOV, A.F. NORJIGITOV
}

\begin{abstract}
Limit theorems in Banach spaces are important, in particular, because of applications in functional data analysis. This paper is devoted to the law of large numbers for the random variables with values in the space $D[0,1]$. This space is not separable if we consider it with supremum norm and it is difficult to prove limit theorems in this space. The law of large numbers is well-studied for the sequences of independent $D[0,1]$-valued random variables. It is known that in the case of independent and identically distributed random variables with values in $D[0,1]$ the existence of the first moment of the norm of random functions is a necessary and sufficient condition for the strong law of large numbers. The law of large numbers for the sequences of independent and not necessarily identically distributed random variables with values in $D[0,1]$ were proved as well. Our main goal is to prove the law of large numbers for the weakly dependent random variables with values in the space $D[0,1]$. Namely, we consider the sequences of mixing random variables with values in $D[0,1]$. Mixing conditions for $D[0,1]$-valued random variables can be introduced in several ways. One can assume that random functions themselves satisfy mixing conditions. We consider a slightly different condition. In fact we assume that the finite dimensional projections of the $D[0,1]$-valued random variables satisfy mixing condition. This is a weaker condition than assuming that random functions themselves satisfy mixing condition. In the paper the law of large numbers for $\rho_{m}$-mixing sequences of $D[0,1]$-valued random variables are proved.
\end{abstract}

Keywords: Law of large numbers, mixing sequence, $D[0,1]$ space.

Mathematics Subject Classification: 60F05, 60D05

\section{INTRODUCTION}

The law of large numbers for sequences of random variables with values in Banach spaces has been investigated by many authors, see [1]-8]. It is known that the validity of the law of large numbers (and also of the strong law of large numbers) depends mainly on the geometry of Banach spaces, see [1], 2], [5], [6] and references therein. The aim of this note is to prove the law of large numbers for weakly dependent random variables with values in $D[0,1]$, which the space of all real-valued functions that are right continuous and have left limits equipped with the norm $\|x(t)\|=\sup _{t \in[0,1]}|x(t)|$. We note that the space $D[0,1]$ is not separable and does not belong to the known types of Banach spaces, see [5].

Let $\left\{X_{n}(t), t \in[0,1], n \geqslant 1\right\}$ be a sequence of $D[0,1]$-valued random variables. We say that the sequence $\left\{X_{n}(t), t \in[0,1], n \geqslant 1\right\}$, with $E X_{k}(t)=0$, satisfies the law of large numbers if

$$
\frac{1}{n}\left(X_{1}(t)+\ldots+X_{n}(t)\right) \rightarrow 0 \text { in probability }
$$

O.Sh. Sharipov, A.F. Norjigitov, LaW of LARge numbers For Weakly Dependent Random VARIABLES With VALUES IN $D[0,1]$.

(C) Sharipov O.Sh., Norjigitov A.F. 2021.

The research of the first author was supported by the Collaborative Research Grant SFB 823 "Statistical modelling of nonlinear dynamic processes" (Germany).

Submitted September 27, 2020. 
as $n \rightarrow \infty$ in $D[0,1]$ and we say that the sequence satisfies the strong law of large numbers if the above convergence holds almost surely.

The laws of large numbers in $D[0,1]$ for the sequences of independent random elements were studied in [9]-[12]. The following theorems were proved in [9]-[11].

Theorem 1.1. 9]. Let $\left\{X_{k}\right\}$ be a sequence of independent and identically distributed random variables with values in $D[0,1]$. Then $\left\{X_{k}\right\}$ satisfies the strong law of large numbers in $D[0,1]$ if and only if $E\left\|X_{1}\right\|<\infty$ and $E X_{1}=0$.

Theorem 1.2. [10]. Let $\left\{X_{n}\right\}$ be a sequence of independent convex dense random elements in $D[0,1]$ satisfying

$$
\sup _{n} E\left\|X_{n}\right\|^{r} \leqslant C,
$$

where $r>1$ and $C$ is a constant. Then, almost surely,

$$
\lim _{n \rightarrow \infty} d\left(n^{-1} \sum_{k=1}^{n} X_{k}, n^{-1} \sum_{k=1}^{n} E X_{k}\right)=0,
$$

where d $(x, y)$ is Skorokhod's metric.

Theorem 1.3. [11. Let $\left\{X_{k}\right\}$ be a sequence of independent random variables with values in $D[0,1]$. Suppose that there exist nondecreasing continuous functions $\varphi$ and $\psi$ on $[0,1]$, such that for all $0 \leqslant s \leqslant t \leqslant u \leqslant 1$, the following conditions hold

$$
\begin{aligned}
& E X_{k}^{2}(t)<\infty \quad \text { for all } t \in[0,1], \quad k=1,2, \ldots \\
& \frac{1}{n^{2}} \sum_{k=1}^{n} E\left\{\left|X_{k}(s)-X_{k}(t)\right|^{2} \wedge\left|X_{k}(t)-X_{k}(u)\right|^{2}\right\} \leqslant \varphi^{2}(u-s), \\
& \int^{\infty} \varphi\left(x^{-2}\right) d x<\infty, \\
& \frac{1}{n^{2}} \sum_{k=1}^{n} E\left\{\left|X_{k}(u)-X_{k}(s)\right|^{2}\right\} \leqslant \psi^{2}(u-s), \\
& \int^{\infty} \psi\left(x^{-4}\right) d x<\infty, \\
& \sum_{k=1}^{\infty} \frac{E\left\|X_{k}\right\|^{p}}{k^{p}}<\infty, \text { for some }, 1 \leqslant p \leqslant 2,
\end{aligned}
$$

where $a \wedge b=\min \{a, b\}$. Then $\left\{X_{n}(t), t \in[0,1], n \geqslant 1\right\}$ satisfies the law of large numbers in $D[0,1]$.

Our aim is to establish the law of large numbers for mixing sequences. Mixing coefficients for a given sequence $\left\{X_{n}(t), t \in[0,1], n \geqslant 1\right\}$ of $D[0,1]$-valued random variables are defined as following:

$$
\begin{aligned}
& \rho(n)=\sup \left\{\frac{|E(\xi-E \xi)(\eta-E \eta)|}{E^{\frac{1}{2}}(\xi-E \xi)^{2} E^{\frac{1}{2}}(\eta-E \eta)^{2}}: \xi \in L_{2}\left(F_{1}^{k}\right), \eta \in L_{2}\left(F_{n+k}^{\infty}\right), k \in \mathbb{N}\right\}, \\
& \rho_{m}(n)=\sup _{\mathbb{R}^{m}} \sup \left\{\frac{|E(\xi-E \xi)(\eta-E \eta)|}{E^{\frac{1}{2}}(\xi-E \xi)^{2} E^{\frac{1}{2}}(\eta-E \eta)^{2}}: \xi \in L_{2}\left(F_{1}^{k}(m)\right), \eta \in L_{2}\left(F_{n+k}^{\infty}(m)\right)\right\},
\end{aligned}
$$

where $F_{a}^{b}$ is a $\sigma$-field generated by random processes $X_{a}(t), \ldots, X_{b}(t), F_{a}^{b}(m)$ is a $\sigma$-field generated by random processes $\prod_{m} X_{a}(t), \ldots, \prod_{m} X_{b}(t)$ and $\prod_{m}: D[0,1] \rightarrow \mathbb{R}^{m}$ is a projection 
operator from $D[0,1]$ to $\mathbb{R}^{m}, L_{2}(F)$ is a space of all square integrable and $F$-measurable random variables.

We say that $\left\{X_{n}(t), t \in[0,1], n \geqslant 1\right\}$ is a $\rho_{m}$-mixing sequence if $\rho_{m}(n) \rightarrow 0$ as $n \rightarrow \infty$ for each $m=1,2, \ldots$ and we say that $\left\{X_{n}(t), t \in[0,1], n \geqslant 1\right\}$ is $\rho$-mixing if $\rho(n) \rightarrow 0$ as $n \rightarrow \infty$. A $\rho$-mixing sequence is always $\rho_{m}$-mixing but in general, $\rho_{m}$-mixing sequence may not be $\rho$-mixing.

We note that we in fact require that the $m$-dimensional projections of the sequence $\left\{X_{n}(t), t \in[0,1], n \geqslant 1\right\}$, which can be denoted by $\left\{X_{n}\left(t_{1}\right), \ldots, X_{n}\left(t_{m}\right), n \geqslant 1\right\}$, satisfy the mixing conditions.

\section{MAIN RESULTS}

The main aim of this work is to prove the following theorems.

Theorem 2.1. Let $\left\{X_{n}(t), t \in[0,1], n \geqslant 1\right\}$ be a sequence of random variables with values in $D[0,1]$. Suppose that there exist a nondecreasing continuous function $H(t)$ on $[0,1]$ such that for all $0 \leqslant s \leqslant u \leqslant 1, n \geqslant 1$ and some $\varepsilon>0$ the following conditions hold:

$$
\begin{aligned}
& E X_{k}(t)=0, E\left|X_{k}(t)\right|^{2}<A \quad \text { for some } A>0, \quad t \in[0,1], \quad k=1,2, \ldots, \\
& \frac{1}{n^{2}} \sum_{k=1}^{n} E\left(X_{k}(u)-X_{k}(s)\right)^{2} \leqslant(H(u)-H(s)) \log ^{-(3+\varepsilon)}\left(1+(H(u)-H(s))^{-1}\right), \\
& \sum_{k=1}^{\infty} \rho_{m}\left(2^{k}\right)<\infty, \quad m=1,2, \ldots
\end{aligned}
$$

Then $\left\{X_{n}(t), t \in[0,1], n \geqslant 1\right\}$ satisfies the law of large numbers in $D[0,1]$.

Theorem 2.2. Let $\left\{X_{n}(t), t \in[0,1], n \geqslant 1\right\}$ be a sequence of random variables with values in $D[0,1]$. Suppose that there exist a nondecreasing continuous function $H(t)$ on $[0,1]$ such that for all $0 \leqslant s \leqslant u \leqslant 1, n \geqslant 1$ and some $\varepsilon>0$ the following conditions hold:

$$
\begin{aligned}
& E X_{k}(t)=0, \quad E\left|X_{k}(t)\right|^{2+\varepsilon}<A, \text { for some } A>0, t \in[0,1], k=1,2, \ldots, \\
& \frac{1}{n^{\frac{2+\varepsilon}{2}}} \max _{1 \leqslant k \leqslant n} E\left|X_{k}(u)-X_{k}(s)\right|^{2+\varepsilon} \leqslant(H(u)-H(s)) \log ^{-(3+2 \varepsilon)}\left(1+(H(u)-H(s))^{-1}\right), \\
& \sum_{k=1}^{n} \rho_{m}^{\frac{2}{2+\varepsilon}}\left(2^{k}\right)<\infty, m=1,2, \ldots
\end{aligned}
$$

Then $\left\{X_{n}(t), t \in[0,1], n \geqslant 1\right\}$ satisfies the law of large numbers in $D[0,1]$.

The following corollaries are immediate consequences of Theorems 2.1, 2.2.

Corollary 2.1. Let $\left\{X_{n}(t), t \in[0,1], n \geqslant 1\right\}$ be a sequence of random variables with values in $D[0,1]$. Suppose that there exist a nondecreasing continuous function $H(t)$ on $[0,1]$ such that for all $0 \leqslant s \leqslant u \leqslant 1$ and some $\varepsilon>0$ the following conditions hold:

$$
\begin{aligned}
& E X_{k}(t)=0, E\left|X_{k}(t)\right|^{2}<A \quad \text { for some } A>0, \quad t \in[0,1], \quad k=1,2, \ldots, \\
& E\left(X_{k}(u)-X_{k}(s)\right)^{2} \leqslant(H(u)-H(s)) \log ^{-(3+\varepsilon)}\left(1+(H(u)-H(s))^{-1}\right), \\
& \sum_{k=1}^{\infty} \rho_{m}\left(2^{k}\right)<\infty, \quad m=1,2, \ldots
\end{aligned}
$$

Then $\left\{X_{n}(t), t \in[0,1], n \geqslant 1\right\}$ satisfies the law of large numbers in $D[0,1]$. 
Corollary 2.2. Let $\left\{X_{n}(t), t \in[0,1], n \geqslant 1\right\}$ be a sequence of random variables with values in $D[0,1]$. Suppose that there exist a nondecreasing continuous function $H(t)$ on $[0,1]$ such that for all $0 \leqslant s \leqslant u \leqslant 1$ and some $\varepsilon>0$ the following conditions hold:

$$
\begin{aligned}
& E X_{k}(t)=0, \quad E\left|X_{k}(t)\right|^{2+\varepsilon}<A \quad \text { for some } A>0, \quad t \in[0,1], \quad k=1,2, \ldots, \\
& E\left|X_{k}(u)-X_{k}(s)\right|^{2+\varepsilon} \leqslant(H(u)-H(s)) \log ^{-(3+2 \varepsilon)}\left(1+(H(u)-H(s))^{-1}\right), \\
& \sum_{k=1}^{n} \rho_{m}^{\frac{2}{2+\varepsilon}}\left(2^{k}\right)<\infty, \quad m=1,2, \ldots
\end{aligned}
$$

Then $\left\{X_{n}(t), t \in[0,1], n \geqslant 1\right\}$ satisfies the law of large numbers in $D[0,1]$.

\section{PROOF OF RESUlTS}

We are going to prove the weak convergence $S_{n}(t) \Rightarrow 0$ as $n \rightarrow \infty$, where

$$
S_{n}(t)=\frac{1}{n} \sum_{k=1}^{n} X_{k}(t) .
$$

This will imply the convergence in probability since in the limit we have a degenerate distribution. We employ the approaches from [14]-[15] used there in the proof of the central limit theorem.

First let us prove that the family of distributions $P_{S_{n}}$ is dense. The proof is based on the following lemmata.

Lemma 3.1. [14]. Let $X_{1}(t), X_{2}(t), \ldots, X_{n}(t), \ldots$ be a random variables with values in $D[0,1]$. Assume that there exist nondecreasing continuous function $H$ on $[0,1]$ and positive numbers $\gamma_{1}, C, \varepsilon$ such that for all $\lambda>0$ and $0 \leqslant s \leqslant t \leqslant u \leqslant 1$

$$
P\left(\left|X_{n}(t)-X_{n}(s)\right| \wedge\left|X_{n}(u)-X_{n}(t)\right| \geqslant \lambda\right) \leqslant C \lambda^{-2 \gamma_{1}} g_{2 \gamma_{1}+1+\varepsilon}(H(u)-H(s))
$$

where $g_{p}(u)=u|\log u|^{-p}, p>0$. Then the family of probability measures $P_{S_{n}}$ is dense.

Lemma 3.2. [13]. Let $\left\{X_{i}, i \geqslant 1\right\}$ be a sequence of real-valued random variables with $\rho$-mixing and for some $q \geqslant 2$

$$
E X_{i}=0, \quad E\left|X_{i}\right|^{q}<\infty, \quad \sum_{k=1}^{n} \rho^{\frac{2}{q}}\left(2^{k}\right)<\infty .
$$

Then there exists a constant $K$ such that the inequality

$$
E\left|X_{1}+\ldots+X_{n}\right|^{q} \leqslant K\left(n^{q / 2} \max _{1 \leqslant i \leqslant n}\left(E X_{i}^{2}\right)^{q / 2}+n \max _{1 \leqslant i \leqslant n} E X_{i}^{q}\right)
$$

holds true.

Proof of Theorem 2.1. The proof is based on Lemma 3.1. It is sufficient to show that

$$
\begin{aligned}
P\left(\left|S_{n}(t)-S_{n}(s)\right| \wedge\left|S_{n}(u)-S_{n}(t)\right| \geqslant \lambda\right) & \leqslant P\left(\left|S_{n}(t)-S_{n}(s)\right|^{2} \wedge\left|S_{n}(u)-S_{n}(t)\right|^{2} \geqslant \lambda^{2}\right) \\
& \leqslant P\left(\left|S_{n}(t)-S_{n}(s)\right|\left|S_{n}(u)-S_{n}(t)\right| \geqslant \lambda^{2}\right) \\
& \leqslant C \lambda^{-2} g_{3+\varepsilon}(H(u)-H(s))
\end{aligned}
$$

where $\lambda \in(0,1], 0 \leqslant s \leqslant t \leqslant u \leqslant 1, \varepsilon>0$.

Note that we can assume $H(u)-H(s) \leqslant \frac{1}{4}$. In what follows, by $C$ we denote various constants, possibly depending on different parameters, that may be different even in the same 
chain of inequalities. We have

$$
\begin{aligned}
J & =\left|S_{n}(t)-S_{n}(s)\right|\left|S_{n}(u)-S_{n}(t)\right| \\
& =\left|\frac{1}{n} \sum_{k=1}^{n}\left(X_{k}(t)-X_{k}(s)\right)\right|\left|\frac{1}{n} \sum_{k=1}^{n}\left(X_{k}(u)-X_{k}(t)\right)\right| \\
& =C\left|\frac{1}{n} \sum_{k=1}^{n}\left(X_{k}(t)-X_{k}(s)\right)\right|^{2}+C\left|\frac{1}{n} \sum_{k=1}^{n}\left(X_{k}(u)-X_{k}(t)\right)\right|^{2}=J_{1}+J_{2} .
\end{aligned}
$$

In the last line, we have used the following notation:

$$
J_{1}=C\left|\frac{1}{n} \sum_{k=1}^{n}\left(X_{k}(t)-X_{k}(s)\right)\right|^{2}, \quad J_{2}=C\left|\frac{1}{n} \sum_{k=1}^{n}\left(X_{k}(u)-X_{k}(t)\right)\right|^{2} .
$$

We have

$$
P\left(J \geqslant \lambda^{2}\right) \leqslant P\left(J_{1} \geqslant \frac{1}{2} \lambda^{2}\right)+P\left(J_{2} \geqslant \frac{1}{2} \lambda^{2}\right) .
$$

Let us estimate each of these terms separately. Using Markov inequality and Lemma 3.2, we get

$$
\begin{aligned}
P\left(J_{1} \geqslant \frac{1}{2} \lambda^{2}\right) & =P\left(\frac{1}{n^{2}}\left|\sum_{k=1}^{n}\left(X_{k}(t)-X_{k}(s)\right)\right|^{2} \geqslant \frac{1}{2} \lambda^{2}\right) \\
& \leqslant 2 C \lambda^{-2} \frac{1}{n^{2}} E\left|\sum_{k=1}^{n}\left(X_{k}(t)-X_{k}(s)\right)\right|^{2} \\
& \leqslant 2 C \lambda^{-2} \frac{1}{n^{2}} \sum_{k=1}^{n} E\left|X_{k}(t)-X_{k}(s)\right|^{2} \\
& \leqslant 2 C \lambda^{-2}(H(t)-H(s)) \log ^{-(3+\varepsilon)}\left(1+(H(t)-H(s))^{-1}\right) .
\end{aligned}
$$

In the same way for $J_{2}$ we get

$$
\begin{aligned}
P\left(J_{2} \geqslant \frac{1}{2} \lambda^{2}\right) & =P\left(\frac{1}{n^{2}}\left|\sum_{k=1}^{n}\left(X_{k}(u)-X_{k}(t)\right)\right|^{2} \geqslant \frac{1}{2} \lambda^{2}\right) \\
& \leqslant 2 C \lambda^{-2}(H(u)-H(t)) \log ^{-(3+\varepsilon)}\left(1+(H(u)-H(t))^{-1}\right) .
\end{aligned}
$$

Hence,

$$
P\left(J \geqslant \lambda^{2}\right) \leqslant 4 C \lambda^{-2}(H(u)-H(s)) \log ^{-(3+\varepsilon)}\left(1+(H(u)-H(s))^{-1}\right) .
$$

By the assumptions of Theorem 2.1 and by the inequality

$$
\log ^{-1}\left(1+(H(u)-H(s))^{-1}\right) \leqslant 2|\log (H(u)-H(s))|^{-1}
$$

for $H(u)-H(s) \leqslant \frac{1}{4}$ we have

$$
\begin{aligned}
& P\left(\left(\left|S_{n}(t)-S_{n}(s)\right| \wedge 1\right)\left(S_{n}(u)-S_{n}(t)\right) \geqslant \lambda^{2}\right) \\
& \quad \leqslant 4 C \lambda^{-2}(H(u)-H(s)) \log ^{-(3+\varepsilon)}\left(1+(H(u)-H(s))^{-1}\right) \\
& \quad \leqslant 4 C \lambda^{-2} g_{3+\varepsilon}(H(u)-H(s)) .
\end{aligned}
$$


In order to complete the proof of the theorem, it remains to prove the convergence of finite dimensional distributions of $S_{n}(t)$. In view of Cramer-Wold theorem [16], to establish this, it is sufficient to prove the law of large numbers for

$$
y_{n}\left(t_{1}, \ldots, t_{k}\right)=\sum_{i=1}^{k} p_{i} X_{n}\left(t_{i}\right)
$$

for each $p_{i} \in \mathbb{R}, i=1,2, \ldots$ and $t_{1}, \ldots, t_{k} \in[0,1]$.

The sequence $\left\{y_{n}\left(t_{1}, \ldots, t_{k}\right), n \geqslant 1\right\}$ satisfies the $\rho_{m}$-mixing condition and by the Chebyshev inequality and the assumptions of Theorem 2.1 and Lemma 3.2 we have

$$
P\left(\left|\frac{1}{n} \sum_{i=1}^{n} y_{i}\left(t_{1}, \ldots, t_{k}\right)\right|>\varepsilon\right) \leqslant \frac{1}{n^{2} \varepsilon^{2}} E\left|\sum_{i=1}^{n} y_{i}\left(t_{1}, \ldots, t_{k}\right)\right|^{2} \leqslant \frac{C n}{n^{2} \varepsilon^{2}} \rightarrow 0,
$$

for all $t_{1}, \ldots, t_{k} \in[0,1], \varepsilon>0, k=1,2, \ldots$ with some constant $C$. The proof is complete.

Proof of Theorem 2.2. We follow the lines of the previous proof. It follows from Lemma 3.1 that it is sufficient to prove

$$
\begin{aligned}
& P\left(\left|S_{n}(t)-S_{n}(s)\right| \wedge\left|S_{n}(u)-S_{n}(t)\right| \geqslant \lambda\right) \\
& \quad \leqslant P\left(\left|S_{n}(t)-S_{n}(s)\right|^{\frac{2+\varepsilon}{2}}\left|S_{n}(u)-S_{n}(t)\right|^{\frac{2+\varepsilon}{2}} \geqslant \lambda^{2+\varepsilon}\right) \\
& \quad \leqslant C \lambda^{-(2+\varepsilon)} g_{3+2 \varepsilon}(H(u)-H(s)),
\end{aligned}
$$

where $\lambda \in(0,1], 0 \leqslant s \leqslant t \leqslant u \leqslant 1, \varepsilon>0$.

We note that we can assume that $H(u)-H(s) \leqslant \frac{1}{4}$. We have:

$$
\begin{aligned}
J & =\left|S_{n}(t)-S_{n}(s)\right|^{\frac{2+\varepsilon}{2}}\left|S_{n}(u)-S_{n}(t)\right|^{\frac{2+\varepsilon}{2}} \\
& =\left|\frac{1}{n} \sum_{k=1}^{n}\left(X_{k}(t)-X_{k}(s)\right)\right|^{\frac{2+\varepsilon}{2}}\left|\frac{1}{n} \sum_{k=1}^{n}\left(X_{k}(u)-X_{k}(t)\right)\right|^{\frac{2+\varepsilon}{2}} \\
& \leqslant C\left|\frac{1}{n} \sum_{k=1}^{n}\left(X_{k}(t)-X_{k}(s)\right)\right|^{2+\varepsilon}+C\left|\frac{1}{n} \sum_{k=1}^{n}\left(X_{k}(u)-X_{k}(t)\right)\right|^{2+\varepsilon} \\
& =C\left|\frac{1}{n} \sum_{k=1}^{n}\left(X_{k}(t)-X_{k}(s)\right)\right|^{2+\varepsilon}+C\left|\frac{1}{n} \sum_{k=1}^{n}\left(X_{k}(u)-X_{k}(t)\right)\right|^{2+\varepsilon}=J_{1}+J_{2} .
\end{aligned}
$$

Here we have used the following notation:

$$
J_{1}=C\left|\frac{1}{n} \sum_{k=1}^{n}\left(X_{k}(t)-X_{k}(s)\right)\right|^{2+\varepsilon}, \quad J_{2}=C\left|\frac{1}{n} \sum_{k=1}^{n}\left(X_{k}(u)-X_{k}(t)\right)\right|^{2+\varepsilon} .
$$

We get:

$$
P\left(J \geqslant \lambda^{2+\varepsilon}\right) \leqslant P\left(J_{1} \geqslant \frac{1}{2} \lambda^{2+\varepsilon}\right)+P\left(J_{2} \geqslant \frac{1}{2} \lambda^{2+\varepsilon}\right) .
$$


We shall estimate each of these terms separately. Using Markov inequality and Lemma 3.2, we obtain

$$
\begin{aligned}
P\left(J_{1} \geqslant \frac{1}{2} \lambda^{2+\varepsilon}\right) & =P\left(\frac{1}{n^{2+\varepsilon}}\left|\sum_{k=1}^{n}\left(X_{k}(t)-X_{k}(s)\right)\right|^{2+\varepsilon} \geqslant \frac{1}{2} \lambda^{2+\varepsilon}\right) \\
& \leqslant 2 C \lambda^{-(2+\varepsilon)} \frac{1}{n^{2+\varepsilon}} E\left|\sum_{k=1}^{n}\left(X_{k}(t)-X_{k}(s)\right)\right|^{2+\varepsilon} \\
& \leqslant 4 C \lambda^{-(2+\varepsilon)}(H(t)-H(s)) \log ^{-(3+2 \varepsilon)}\left(1+(H(t)-H(s))^{-1}\right) .
\end{aligned}
$$

In the same way, for $J_{2}$ we get

$$
\begin{gathered}
P\left(J_{2} \geqslant \frac{1}{2} \lambda^{2+\varepsilon}\right) \leqslant 4 C \lambda^{-(2+\varepsilon)}(H(u)-H(t)) \log ^{-(3+2 \varepsilon)}\left(1+(H(u)-H(t))^{-1}\right), \\
P\left(J \geqslant \lambda^{2+\varepsilon}\right) \leqslant 8 C \lambda^{-(2+\varepsilon)}(H(u)-H(s)) \log ^{-(3+2 \varepsilon)}\left(1+(H(u)-H(s))^{-1}\right) .
\end{gathered}
$$

By the assumptions of Theorem 2.2 and by the inequality

$$
\log ^{-1}\left(1+(H(u)-H(s))^{-1}\right) \leqslant 2|\log (H(u)-H(s))|^{-1}
$$

for $H(u)-H(s) \leqslant \frac{1}{4}$, as in the proof of previous theorem, we find:

$$
\begin{aligned}
P & \left(\left|S_{n}(t)-S_{n}(s)\right|^{\frac{2+\varepsilon}{2}}\left|S_{n}(u)-S_{n}(t)\right|^{\frac{2+\varepsilon}{2}} \geqslant \lambda^{2+\varepsilon}\right) \\
& \leqslant 4 C \lambda^{-(2+\varepsilon)}(H(u)-H(s)) \log ^{-(3+2 \varepsilon)}\left(1+(H(u)-H(s))^{-1}\right) \\
& \leqslant 4 C \lambda^{-(2+\varepsilon)} g_{3+2 \varepsilon}(H(u)-H(s)) .
\end{aligned}
$$

This proves the required density. The convergence of finite-dimensional distributions of $S_{n}(t)$ follows from Cramer-Wold theorem [16] as in the proof of Theorem 2.1. The proof is complete.

\section{ACKNOWLEDGMENTS}

The authors thank an anonymous referee for a valuable report.

\section{REFERENCES}

1. D. Li, Y. Qi and A. Rosalsky. A refinement of the Kolmogorov-Marcinkiewicz-Zygmund strong law of large numbers // J. Theor. Prob. 24:4, 1130-1156 (2011).

2. A. De Acosta. Inequalities for B-valued random vectors with applications to the law of large numbers // Ann. Prob. 9:1, 157-161 (1981).

3. F. Hechner and B. Heinkel. The Marcinkiewicz-Zygmund LLN in Banach spaces. A generalized martingale approach // J. Theor. Prob. 23:2, 509-522 (2010).

4. J. Hoffmann-Jorgensen and G. Pisier. The law of large numbers and the central limit theorem in Banach spaces // Ann. Prob. 4:4, 587-599 (1976).

5. M. Ledoux and M. Talagrand. Probability in Banach spaces: Isoperimetry and processes. Springer, Berlin (1991).

6. G. Pisier. Probabilistic methods in the geometry of Banach spaces // In: "Probability and Analysis". Springer, Berlin. 1206, 167-241 (1986).

7. J. Rosinski. Remarks on Banach spaces of stable type // Prob. Math. Stat. 1:1, 67-71 (1980).

8. R.L. Taylor. Stochastic convergence of weighted sums of random elements in linear spaces. Springer, Berlin (1978).

9. R. Ranga Rao. The law of large numbers for $D[0,1]$-valued random variables // Theor. Prob. Appl. 8:1, 70-74 (1963).

10. P.Z. Daffer and R.L. Taylor. Laws of large numbers for $D[0,1] / /$ Ann. Prob. 7:1, 85-95 (1979). 
11. P. Bezandry. Laws of large numbers for $D[0,1] / /$ Stat. Prob. Lett. 76:10, 981-985 (2006).

12. J. Kuelbs and J. Zinn. Some stability results for vector valued random variables // Ann. Prob. 7:1, 75-84 (1979).

13. Q. Shao. Maximal inequalities for partial sums of $\rho$-mixing sequences // Ann. Prob. 23:2, 948-965 (1995).

14. M. Bloznelis and V. Paulauskas. On the central limit theorem in the space $D[0,1] / /$ Stat. Prob. Lett. 17:2, 105-111 (1993).

15. V. Paulauskas and Ch. Stieve. On the central limit theorem in $D[0,1]$ and $D([0,1], H) / /$ Liet. Matem. Rink. 30, 567-579 (1990).

16. P. Billingsley. Convergence of probability measures. Wiley, New York (1968).

Olimjon Shukurovich Sharipov,

National University of Uzbekistan

named after Mirzo Ulugbek,

100174 University street 4,

Almazar district, Tashkent, Uzbekistan

E-mail: osharipov@yahoo.com

Anvar Fayzullayevich Norjigitov,

V.I. Romanovskiy Institute of Mathematics,

Academy of Sciences of Uzbekistan,

100174 University street 4b,

Almazar district, Tashkent, Uzbekistan

E-mail: anvar2383@mail.ru 